\title{
Preparation of supported-deep eutectic solvent membranes: Effects of bath medium composition on the structure and performance of supported-deep eutectic solvent membrane for $\mathrm{CO}_{2} / \mathrm{N}_{2}$ gas separation
}

\author{
Amira Mohd Nasib a, ${ }^{*}$, Irfan Hatim a, Nora Jullok a, Syahmie Rasidi ${ }^{b}$ \\ a School of Bioprocess Engineering, Universiti Malaysia Perlis, 02600 Arau, Perlis, Malaysia \\ b School of Material Engineering, Universiti Malaysia Perlis, 02600 Arau, Perlis, Malaysia \\ * Corresponding author: amira.m.nasib@gmail.com
}

\section{Article history}

Received 10 Mac 2019

Revised 31 May 2019

Accepted 3 February 2020

Published Online 15 June 2020

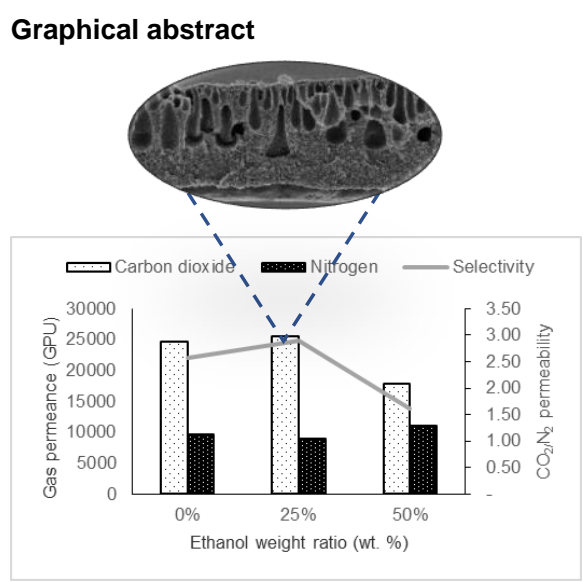

\begin{abstract}
Polyvinylidene fluoride-co-polytetrafluoroethylene, PVDF-co-PTFE polymer was used as a membrane support. The asymmetric membranes were formed by immersion of casted membrane film into the coagulation bath. This work manipulated the coagulant bath medium by mixing ethanol with distilled water at different weight percentages $(0,25$ and 50 wt. \% of ethanol). The structures of fabricated membranes were observed to have different morphologies. Higher ethanol content altered the membrane structure from finger-like to sponge-like structure, and hence differed in membrane porosity. Vacuum-based technique was chosen to impregnate the deep eutectic solvent (DES) into the pores of membrane support. DES was prepared by mixing choline chloride $(\mathrm{ChCl})$ and ethylene glycol at a ratio of 1:3. Scanning electron microscopy (SEM) was used to study the membrane morphology changes while in order to determine the immobilization of DES, energy dispersive X-ray (EDX) analysis was used. The porosity of fabricated PVDF-co-PTFE membrane was determined by means of gravimetric method. Lastly, the membrane separation performance using $\mathrm{CO}_{2}$ and $\mathrm{N}_{2}$ gasses were used to determine the capability of the supported-DES-membrane. The results demonstrated the highest immobilization of DES in supported membrane pores was achieved when combination of $25 \mathrm{wt}$. \% of ethanol and $75 \mathrm{wt} . \%$ distilled water was used as a coagulant bath medium. The respective membrane has $74.5 \%$ porosity with the most excellent performance of $\mathrm{CO}_{2}$ separation at $25.5 \times 10^{3} \mathrm{GPU}$ with $\mathrm{CO}_{2} / \mathrm{N}_{2}$ selectivity of 2.89 .
\end{abstract}

Keywords: PVDF-co-PTFE polymer, coagulant bath medium, gas separation, deep eutectic solvents

\section{INTRODUCTION}

Carbon dioxide $\left(\mathrm{CO}_{2}\right)$ is one of the greenhouse gases other than water vapor, methane, nitrous oxide and ozone. Extreme emission of $\mathrm{CO}_{2}$ to the atmosphere is believed to cause a global warming effect. If the $\mathrm{CO}_{2}$ gas concentration released to the atmosphere keeps increasing, the gas concentration may reach up to $570 \mathrm{ppm}$ by a year of 2100, as predicted by International Panel on Climate Change (IPCC). This will then lead to increasing of mean sea level by $38 \mathrm{~m}$ and increasing of global mean temperature by $1.9{ }^{\circ} \mathrm{C}$, which may cause a negative impact to the ecosystem, biodiversity and human activities $[1,2]$. There are few technologies available to capture $\mathrm{CO}_{2}$ gas such as physical and chemical absorption, adsorption, cryogenic distillation and membrane separation. Up to date, cryogenic distillation and absorption technology using aqueous alkanoamines solutions have been applied in industrial level and used commercially [3]. Nevertheless, both technologies possess few drawbacks such as high possibility of equipment corrosion, high in energy consumption and requirement of larger equipment [4]. In recent years, membrane separation has become an attractive technology due to its low in both capital and operating cost, simple operation and lower energy requirements. The advantages of membrane technology are believed $\bar{a}$ to overcome most of the traditional method problems.

Polymer based membrane has been widely used in industrial level for $\mathrm{CO}_{2}$ separation. Cellulose acetate, polyimides, polysulfone, polycarbonate and polyamides are examples of the polymer materials employed as a membrane precursor [5]. However, current polymer membranes possess few disadvantages such as plasticization, aging and also permeability-selectivity trade-off [6]. Thus, supported liquid membrane (SLM) has been introduced and explored in the last 15 years to overcome commercial polymer solid membrane drawbacks [1]. Basically, liquid in SLM is retained in the membrane pores by capillary forces. However, SLM is not yet been applied in high scale due to instability of liquid in membrane supports. The stability of SLM can be affected by few factors such as polymer support chemical natures and morphology properties, type of chosen liquid and method of SLM preparation [7]. 
The liquid chosen must has a certain criteria to develop a stable SLM such as low votality, hydrophobic, moderate viscosity and high surface tension $[8,9]$. The research using ionic liquids (IL) in SLM has become an attractive solvent since it has been introduced by Blanchard and co-workers for $\mathrm{CO}_{2}$ separation in 2001 [1]. ILs are molten salts, liquid at room temperature and possess a tuneable physicochemical properties like their viscosity, density, hydrophilicity and solubility. Despite of their advantages, the environmental friendliness of some ILs are still uncertain due to their poor biodegradability, biocompatibility and sustainability. In addition, synthesis of ILs is costly and high energy demand for recycling purposes. [10-12]. Hence, a new solvent named DES has been introduced to overcome the ILs toxicity and cost.

DES is prepared by mixing two or three safe components that interact via hydrogen bonding to form an eutectic mixture. The properties of DES are similar to ILs in such DES contains mostly of ionic species, which favour high dissolution of $\mathrm{CO}_{2}$ [13]. This characteristic is believed can be a good potential in chemical processes specifically in $\mathrm{CO}_{2}$ gas separation and purification. DES was firstly synthesized by mixing choline chloride as a hydrogen bond acceptor and urea as a hydrogen bond donor [14]. Impregnation of DES into the pores of the polymer membrane is still new and not yet been reported. Thus, it is crucial to understand the behaviour of DES in the membrane support.

The aim of this work was to investigate the effect of coagulant bath medium on DES loading in the membrane pores and the SLM performance for $\mathrm{CO}_{2} / \mathrm{N}_{2}$ gas separation. Thus, this work was carried out by preparing PVDF-co-PTFE membrane supports via phase inversion method. The coagulant bath medium was manipulated at different weight ratios of ethanol and distilled water. DES was synthesized by mixing of ethylene glycol and choline chloride, before being impregnated into the membrane pores using vacuum method.

\section{EXPERIMENTAL}

\section{Materials}

PVDF-co-PTFE polymer was obtained from Arkema Pte Ltd, Singapore. Ethylene glycol and N,N-dimethylacetamide (DMAc) were bought from Merck Milipore Corporation. Meanwhile, polyethylene glycol (PEG) with an average MW 10,000 was supplied by Sigma Aldrich. Lastly, ethanol (EtOH) was purchased from $\mathrm{HmbG}^{\circledR}$ Chemicals. All chemicals used were $\geq 99.0 \%$ purity.

\section{Preparation of membrane support}

A 77 wt. \% of DMAc solvent was used to dissolve 20 wt. \% of PVDF-co-PTFE polymer in a closed conical flask under continuous rotation for 2 hour using an overhead stirrer. Later, PEG at 3 wt. \% was added and the stirring was continued until a homogenous solution was obtained. The temperature of the solution was preserved at $50{ }^{\circ} \mathrm{C}$. Then, the final polymer solution was aerated to remove the air bubbles in an oven with a setting of $45 \pm 5{ }^{\circ} \mathrm{C}$ for 24 hours. After aeration, the polymer solution was hand-casted using a hand-casting knife on a glass plate. The gap of casting knife was set at $400 \pm 10 \mu \mathrm{m}$. Next, the casted film was exposed to the surrounding air for 30 seconds before submerging into a coagulation bath for 24 hours to allow the phase inversion process [15]. Table 1 lists the ratio of ethanol and distilled water used in coagulant bath medium. Lastly, the solidified membrane was removed from the coagulation bath and dried for 24 hours at ambient temperature.

Table 1 Conditions of coagulant bath medium.

\begin{tabular}{ccc}
\hline & \multicolumn{2}{c}{$\begin{array}{c}\text { Coagulant bath medium (wt. } \\
\text { \%) }\end{array}$} \\
\cline { 2 - 3 } Membrane & Ethanol & $\begin{array}{c}\text { Distilled } \\
\text { water }\end{array}$ \\
\cline { 2 - 3 } & & 100 \\
M-0E & 0 & 75 \\
M-25E & 25 & 50 \\
M-50E & 50 & \\
\hline
\end{tabular}

\section{Supported-DES-membranes preparation}

Ethylene glycol and choline chloride $(\mathrm{ChCl})$ with a ratio of $3: 1$ were mixed together to synthesize DES. At $200 \mathrm{rpm}$, the mixture was stirred continuously at room temperature until the solution became clear and homogenous.

Vacuum immobilization technique was applied to prepare the supported membranes in this study. The synthesized membrane was totally immersed into DES in a petri dish and then placed in a desiccator at room temperature. The pressure of desiccator was set at 10 mbar using a vacuum pump for 3 hours. In order to ensure all the membrane pores were filled up with the solvent, the desiccator was aerated for every hour. Then, by using a filter paper, the excess DES on the membrane surface was wiped off.

Finally, the membrane was coated with 3 wt. \% PDMS/n-hexane solution to seal the pinholes and maintain the solvent in the membrane pores. Coating procedure was accomplished by totally immersing the membrane in PDMS solution for 10 minutes at room temperature before being dried for 24 hours at room temperature.

\section{Membrane characterization}

Scanning electron microscope (SEM) with model of JSM 6260 LE JEOL was used to investigate the morphologies of synthesized membrane top surface and the membrane cross sectional area. The membrane pore sizes were measured from the SEM images by using ImageJ software. Meanwhile, the overall chemical composition and chemical elements distribution in the membrane pores were analysed by additional of energy-dispersive X-ray (EDX).

The membrane porosity was determined by using gravimetric method. The sample was immersed in octanol for 30 seconds. Then, the sample was dried using a filter paper. Sample mass of before and after soaking procedure were recorded. The membrane porosity was calculated by using Equation (1).

$$
\omega=\frac{m_{n} / \rho_{n}}{m_{n} / p_{n}+{ }^{m_{p}} / \rho_{p}}
$$

Where $\omega$ is the membrane porosity, $m_{n}$ is the mass of absorbed octanol, $\rho_{\mathrm{n}}$ is the density of octanol, $\mathrm{m}_{\mathrm{p}}$ is the mass of dry membrane, and $\rho_{p}$ is the density of the membrane. This method was used to estimate the porosity by determining the weight of liquid that contained in the membrane pores.

\section{Gas permeation test}

The supported-DES-membrane performance was tested using pure gas permeation procedure. The pure gas permeance was determined by a constant volume. The membrane was placed in a permeation cell and exposed to pure $\mathrm{CO}_{2}$ or $\mathrm{N}_{2}$ individually. The effective area of permeation cell was $11.34 \mathrm{~cm}^{2}$. Both transmembrane pressure and temperature were constant throughout the testing at $3.5 \mathrm{psi}$ and $27^{\circ} \mathrm{C}$, respectively.

The permeance (pressure-normalized flux) of individual gas was calculated as follows :

$$
\left(\frac{P_{i}}{l}\right)=\frac{1}{A \Delta p} \times \frac{d V_{i}}{d t}
$$

Where $\mathrm{i}$ represents the gas penetrant, $\mathrm{V}_{\mathrm{i}}$ is the volume of the gas permeated through the membrane $\left(\mathrm{cm}^{3}, \mathrm{STP}\right), \mathrm{A}$ is the effective membrane area $\left(\mathrm{cm}^{2}\right), \mathrm{t}$ is the permeation time (s) and $\Delta \mathrm{p}$ is the transmembrane pressure drop $(\mathrm{cmHg})$. The permeance is expressed in gas permeation unit, GPU, as

$G P U=1 \times 10^{-6} \frac{\mathrm{cm}^{3}(S T P)}{\mathrm{cm}^{2} . S . c m H g}$

Meanwhile, the selectivity was obtained by

$$
\alpha_{i / j}=\frac{(P / l)_{i}}{(P / l)_{j}}
$$


Where $\alpha_{(\mathrm{i} j \mathrm{j})}$ is the selectivity of gas i over gas $\mathrm{j}$. Both $(P / l)_{\mathrm{i}}$ and $(P / l)_{\mathrm{j}}$ are the permeance of gas i and $\mathrm{j}$, respectively.

\section{RESULTS AND DISCUSSION}

\section{Effects of coagulant bath medium on membrane morphologies}

Fig. 1 shows the SEM micrographs of (a) the membrane top surface and (b) the cross sectional area with a magnification of 5,000x and 500x, respectively. From Fig 1(a), all three membranes showed clear pores or pinholes which were developed due to the existence of PEG (3 wt. \%) as a pore performer. However, the pores quantities and sizes were differed from each other due to different ethanol contents in the coagulation bath medium. Highest pore formation could be seen from M-0E membrane top surface, whereas both M-25E and M-50E membranes possessed almost the same quantity of pores but variety in size. Among all three membranes, M-50E membrane had a larger pore size on the surface with 50 wt $\%$ ethanol in the coagulant bath medium.

Cross sectional area of $\mathrm{M}-\mathrm{OE}$ membrane synthesized without ethanol in a coagulant bath medium displayed a dominant of macrovoid finger-like structures as shown in Fig 1(b). On the other hand, additional of ethanol by 25 and 50 wt. \% diminished the macrovoid and developed a sponge-like structure as shown by $\mathrm{M}-25 \mathrm{E}$ and M-50E membranes, respectively. Formation of larger finger-like macrovoids structure was due to fast precipitation rate in coagulant bath medium. Meanwhile, sponge-like structure was resulted from slower precipitation rate. In short, the polymer precipitation rate in a coagulant bath medium reduced by the presence of ethanol. Similar observation was obtained by Deshmukh and Li (1998) where the additional of ethanol changed the long finger-like to sponge-like structure of PVDF hollow fiber membranes [16].
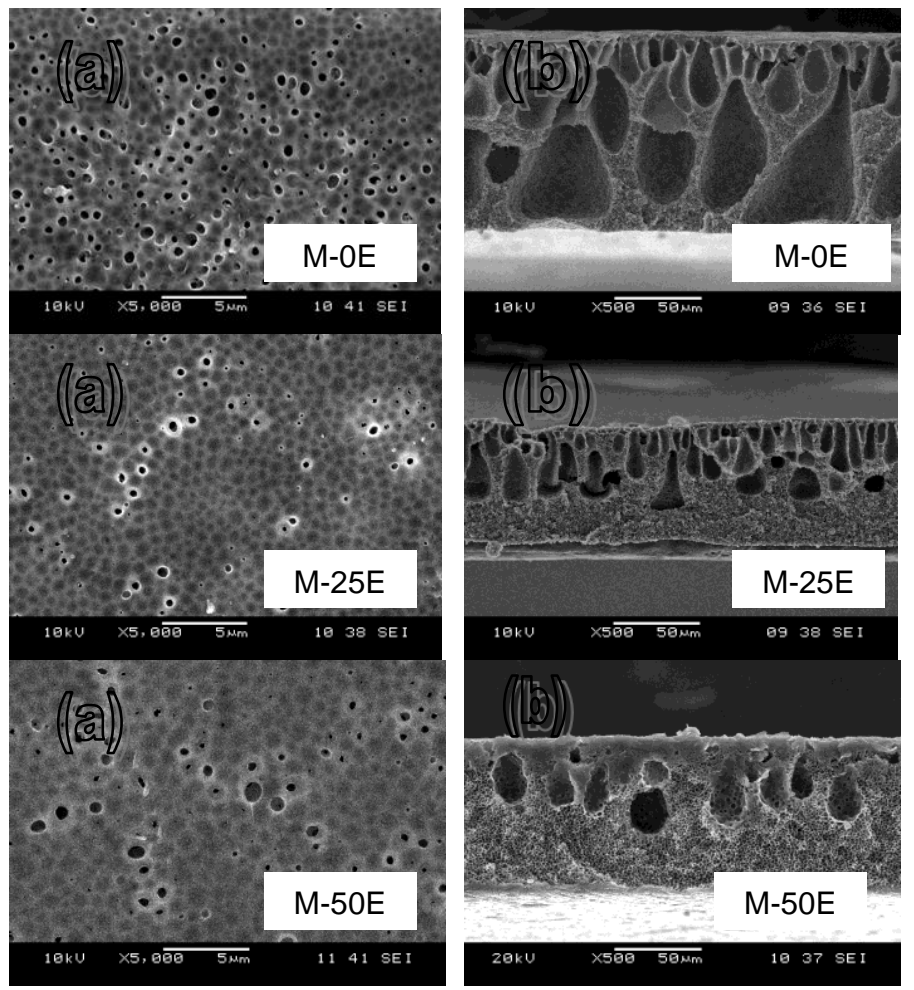

Fig. 1 Micrographs of empty PVDF-co-PTFE membrane (a) membrane top surface and (b) cross sectional view.

Membrane pore size and porosity are among the crucial factors that affect the DES immobilization. The membrane pore sizes were measured from SEM micrographs using ImageJ software. Meanwhile, the membrane porosity was calculated by using gravimetric method. Approximately, 10 readings were repeatedly measured to get the final result. Table 2 lists the synthesized membranes pore size and porosity. The larger pore size between 0.6 to $35.0 \mu \mathrm{m}$ was observed by M-0E membrane which used purely distilled water as a coagulant bath. As the ethanol ratio was increased, the minimum pore sizes were decreased with a trend; $\mathrm{M}-50 \mathrm{E}$ had the smallest minimum pore size, followed by $\mathrm{M}-25 \mathrm{E}$ and $\mathrm{M}-0 \mathrm{E}$ membrane, respectively. $\mathrm{M}-25 \mathrm{E}$ membrane possessed the highest porosity which was $73 \%$. This could be explained by the lowest range between minimum and maximum pore size of the membrane. Among all three membranes, finger-like structure of M-25E showed almost uniformly in size.

Table 2 Membranes pore size and porosity

\begin{tabular}{|c|c|c|c|}
\hline \multirow{2}{*}{ Sample } & \multicolumn{2}{|c|}{ Pore size $(\mu \mathrm{m})$} & \multirow{2}{*}{ Porosity, \% } \\
\hline & Minimum & Maximum & \\
\hline M-OE & 0.6 & 35.0 & 70.5 \\
\hline$M-25 E$ & 0.6 & 18.3 & 73.0 \\
\hline$M-50 E$ & 0.4 & 21.0 & 61.9 \\
\hline
\end{tabular}

Further information on the elemental composition of the samples could be provided by EDX analysis. The elemental composition of supported-DES-membranes is summarized in Table 3. The EDX results confirmed the presence of chlorine, nitrogen and oxygen elements in the samples which corresponded to DES. Both carbon and fluorine elements represented PVDF-co-PTFE membrane support, while PDMS coating could be detected by silicone element. M-25E membrane demonstrated the highest DES loading in the membrane pores for about 55\% DES elements. Meanwhile, M-50E showed the lowest DES loading with only 13\%. This could be related with membrane porosity. As the membrane porosity was increased, DES loading in the membrane pores was also increased with a sequence of $\mathrm{M}-50 \mathrm{E}<\mathrm{M}-0 \mathrm{E}<\mathrm{M}-25 \mathrm{E}$.

Table 3 Elemental analysis by EDX for supported-DES-membranes.

\begin{tabular}{cccc}
\hline & \multicolumn{3}{c}{ Mass \% } \\
\cline { 2 - 4 } Component & M-0E & M-25E & M-50E \\
\hline Chlorine & 21.30 & 19.11 & 8.44 \\
Nitrogen & 20.63 & 30.64 & 5.12 \\
Oxygen & 10.35 & 5.01 & - \\
Carbon & 30.52 & 20.94 & 39.37 \\
Fluorine & 18.49 & 33.40 & 45.93 \\
Silicone & 0.71 & 0.90 & 1.15 \\
\hline
\end{tabular}

\section{Evaluation of supported-DES-membrane performance}

Supported-DES-membrane performance was tested by applying single gas permeation using pure $\mathrm{CO}_{2}$ and $\mathrm{N}_{2}$ at 1 bar pressure and ambient temperature. The permeance and selectivity for M-0E, M-25E and M-50E membranes are displayed in Fig. 2. As presented in Fig. 2, $\mathrm{M}-25 \mathrm{E}$ has a superior performance compared to the other membranes. The permeance of $\mathrm{CO}_{2}$ and $\mathrm{N}_{2}$ recorded for a membrane without presence of ethanol in the coagulant bath was $24.6 \times 10^{3} \mathrm{GPU}$ and 9.6 $x 10^{3} \mathrm{GPU}$, respectively. The permeance of $\mathrm{CO}_{2}$ gas was increased when 25 wt. \% ethanol was added with a value of $25.5 \times 10^{3} \mathrm{GPU}$ while $\mathrm{N}_{2}$ was decreased $\left(8.8 \times 10^{3} \mathrm{GPU}\right)$. However, when the weight ratio of ethanol was further increased to $50 \%$, the $\mathrm{CO}_{2}$ permeance showed a decrement about $40 \%$. The $\mathrm{CO}_{2} / \mathrm{N}_{2}$ selectivity for $\mathrm{M}-25 \mathrm{E}$ membrane was the highest with 2.89 compared to $\mathrm{M}-0 \mathrm{E}$ and $\mathrm{M}-50 \mathrm{E}$ membranes. 


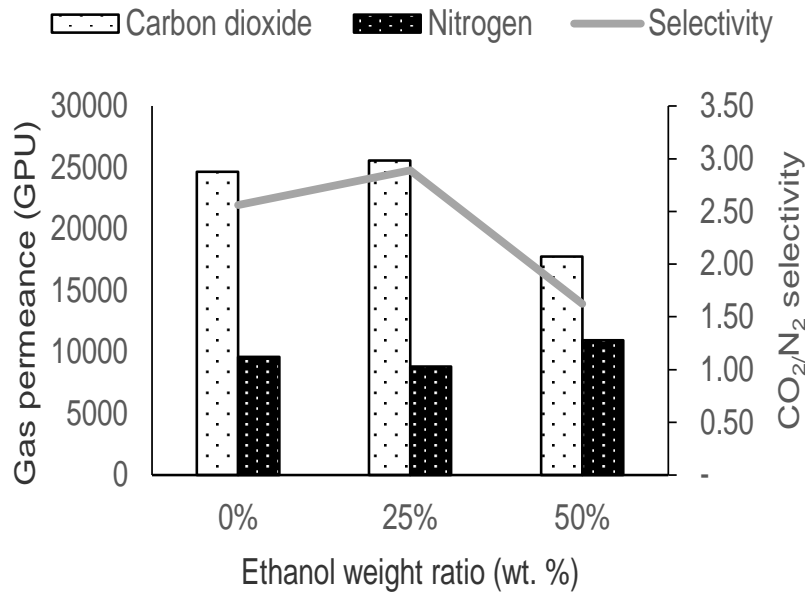

Fig. 2 The permeance and selectivity of supported-DES-membranes.

\section{CONCLUSION}

Supported DES in PVDF-co-PTFE flat sheet membrane using different coagulant bath medium has been successfully synthesized. Presence of ethanol in the bath medium altered the membrane microstructure which proven by SEM micrograph. Moreover, EDX analysis confirmed that combination of finger and sponge-like structures that gave the highest porosity and was able to hold DES in higher quantity. Finally, the performance of supported-DESmembrane was tested via gas permeation test. The results of $\mathrm{CO}_{2}$ permeance and $\mathrm{CO}_{2} / \mathrm{N}_{2}$ selectivity demonstrated that better performance could be achieved when higher DES immobilized in the membrane pore support.

\section{ACKNOWLEDGEMENT}

This work was financially supported by the Universiti Malaysia Perlis (UniMAP) under the Shortterm Grant (9001-00547) and Ministry of Higher Education Malaysia (MOHE).

\section{REFERENCES}

[1] Dai, Z., Noble, R. D., Gin, D. L., Zhang, X., Deng, L. 2016. Combination of ionic liquids with membrane technology: A new approach for $\mathrm{CO}_{2}$ separation. Journal of Membrane Science, 497, 1-20.

[2] Yang, H., Xu, Z., Fan, M., Gupta, R., Slimane, R. B., Bland, A. E., Wright, I. 2008. Progress in carbon dioxide separation and capture: A review. Journal of Environmental Sciences, 1, 20, 14-27.

[3] Leung, D. Y., Caramanna, G., Maroto-Valer, M. M. 2014. An overview of current status of carbon dioxide capture and storage technologies. Renewable and Sustainable Energy Reviews, 39, 426-443.

[4] Yu, C.-H., Huang, C.-H., Tan, C.-S. 2012. A review of CO2 capture by absorption and adsorption. Aerosol and Air Quality Research, 5, 12, 745-769.

[5] Dortmundt, D., Doshi, K. 1999. Recent developments in $\mathrm{CO}_{2}$ removal membrane technology. UOP LLC. 1-30.

[6] Adewole, J., Ahmad, A., Ismail, S., Leo, C. 2013. Current challenges in membrane separation of $\mathrm{CO} 2$ from natural gas: A review. International Journal of Greenhouse Gas Control. 17, 46-65.

[7] Hernández-Fernández, F. J., de los Rios, A. P., Tomás-Alonso, F., Palacios, J. M., Víllora, G. 2012. Understanding the influence of the ionic liquid composition and the surrounding phase nature on the stability of supported ionic liquid membranes. AIChE Journal. 2, 58, 583-590.

[8] Dżygiel, P. and Wieczorek, P. P., Supported liquid membranes and their modifications: definition, classification, theory, stability, application and perspectives, in Liquid Membranes. 2010, Elsevier. p. 73-140.

[9] Ghoshal, A. K., Saha, P., Liquid-membrane filters, in Progress in Filtration and Separation. 2015, Elsevier. p. 155-205.

[10] Paiva, A., Craveiro, R., Aroso, I., Martins, M., Reis, R. L., Duarte, A. R. C. 2014. Natural deep eutectic solvents-solvents for the 21st century. ACS Sustainable Chemistry \& Engineering, 5, 2, 1063-1071.

[11] Zhang, Q., Vigier, K. D. O., Royer, S., Jerome, F. 2012. Deep eutectic solvents: syntheses, properties and applications. Chemical Society Reviews, 21, 41, 7108-7146.

[12] Wang, J., Luo, J., Feng, S., Li, H., Wan, Y., Zhang, X. 2016. Recent development of ionic liquid membranes. Green Energy \& Environment. $1,1,43-61$.

[13] Ali, E., Hadj-Kali, M. K., Mulyono, S., Alnashef, I., Fakeeha, A., Mjalli, F., Hayyan, A. 2014. Solubility of $\mathrm{CO}_{2}$ in deep eutectic solvents: experiments and modelling using the Peng-Robinson equation of state. Chemical Engineering Research and Design, 10, 92, 1898-1906.

[14] Li, G., Deng, D., Chen, Y., Shan, H., Ai, N. 2014. Solubilities and thermodynamic properties of $\mathrm{CO} 2$ in choline-chloride based deep eutectic solvents. The Journal of Chemical Thermodynamics, 75, 58-62.

[15] Ooi, B., Yatim, N., Ahmad, A., Lai, S. 2012. Preparation of polyvinylidene fluoride membrane via dual coagulation bath system and its wettability study. Journal of Applied Polymer Science, S1, 124, E225-E232.

[16] Deshmukh, S., Li, K. 1998. Effect of ethanol composition in water coagulation bath on morphology of PVDF hollow fibre membranes. Journal of Membrane Science, 1, 150, 75-85. 